\title{
Central Venous Pressure as a Predictor of Fluid Responsiveness: Is This All You Need?
}

\author{
Marcello Guarnieri, Alessandro Belletti, Francesco Saglietti, and Elena Bignami
}

Department of Anesthesia and Intensive Care, IRCCS San Raffaele Scientific Institute, Via Olgettina 60, 20132 Milan, Italy

"Corresponding author: Bignami E, Department of Anesthesia and Intensive Care, IRCCS San Raffaele Scientific Institute, Via Olgettina 60,20132 Milan, Italy, Tel: +39 022643 4524; Fax: +39 022643 7178; E-mail: bignami.elena@hsr.it

Rec date: March 08, 2016; Acc date: April 04, 2016; Pub date: April 12, 2016

Copyright: ( 2016 Guarnieri M, et al. This is an open-access article distributed under the terms of the Creative Commons Attribution License, which permits unrestricted use, distribution, and reproduction in any medium, provided the original author and source are credited.

\begin{abstract}
Fluid administration during and after surgery is a fundamental strategy for maintaining hemodynamic stability. Technology offers different strategies for hemodynamic monitoring and decision making about fluids administration. In particular patients may be divided into fluid-responding patients and non-fluid-responding patients. Central venous pressure, measured by a central venous catheter, is a basic measure of right heart preload, and many trials defined it as a non-trustable parameter, with many biases and confounding factors, such as mechanical ventilation and intrathoracic pressure. Nevertheless, many authors describe how a simple value like the Central venous pressure may be useful in hemodynamic monitoring during and after cardiac surgery, although it is not the best available. Moreover the results of a recent survey performed in cardiothoracic intensive care units in Italy, reported how central venous pressure was the easiest and the most frequently used parameter for the monitoring of fluid therapy.
\end{abstract}

Given this state of the art, Central venous pressure seems to be the most criticized but, due to its feasibility and availability, it is still the most used parameter for the evaluation of preload and for the prediction of fluid responsiveness in operating room and Intensive Care Unit.

Keywords: Fluid responsiveness; Perioperative care; Critical care; Central venous pressure

\section{Introduction}

Volume expansion through fluid administration is one of the simplest approaches initial treatments of hemodynamic instability. According to the Frank-Starling Mechanism, an increase in left ventricular preload leads to an increase in cardiac output, as long as we are on the ascending part of the Frank-Starling curve [1]. Nevertheless, excessive fluid administration has recently been shown to be detrimental [2], and anesthesiologists as well as critical care physicians continuously struggle to determine whether or not their patients will benefit from volume expansion.

Due to technological advances, nowadays physicians are offered a wide range of indices and monitoring tools which can be used to assess fluid responsiveness [3]. As a consequence, numerous studies have been carried out to investigate this issue, with considerable debate as to which index should be used. However, there is a good degree of consensus on the fact that central venous pressure (CVP), one of the oldest indices used to monitor volemia, is neither a good parameter to assess volume status nor a good predictor of fluid responsiveness [4], in contrast with more recently developed dynamic indices [5]. Nevertheless, in a recent survey performed in cardiothoracic intensive care units (ICUs) in Italy, CVP was reported to be the parameter most frequently used to monitor fluid therapy in critically ill patients and during the perioperative period in cardiac surgery [6]. Similar findings have been reported in a consistent number of studies performed in different parts of the world in both adult and pediatric patients [7-9]. Although practice might be changing, particularly in the general ICU setting [10], it seems that there is still a relevant gap between research and everyday clinical practice. This might be due to various factors. The easiest explanation is that while dynamic indices were introduced relatively recently, CVP has been used for decades. Changing a practice that has been routine for such a long time is not simple, unless trials with striking results in favor of the new technique (e.g. ventilation with low tidal volumes in acute respiratory distress syndrome) are published. Another explanation might be that dynamic indices too have certain limitations that could become relevant outside the strict control of clinical trials $[11,12]$. For example, they require sinus rhythm, mechanical ventilation with relatively high tidal volumes, a closed chest, and the absence of right ventricular dysfunction, which might limit their use, particularly in cardiothoracic surgery, a setting where accurate fluid optimization is even more critical. In addition, critically ill patients and high-risk perioperative patients, where one might most need monitoring of fluid responsiveness, usually require a central venous catheter for the administration of fluids and drugs, and adding a transducer to measure CVP is easier, less expensive, and less invasive than other devices, such as pulmonary artery catheters (PAC). Furthermore, CVP can also be measured in normal wards without the need for long or complex training. Finally, critical use of CVP provides useful information even for an expert clinician. For example, CVP variations are more relevant than its absolute value. A sudden increase in CVP, together with hypotension, in a patient who recently underwent major surgery might indicate conditions that require immediate attention and treatment such as cardiac tamponade, tension pneumothorax or pulmonary embolism, and trigger further diagnostic evaluation with echocardiography or other imaging, or advanced monitoring to obtain a correct diagnosis and initiate treatment.

To summarize, despite its limitations CVP is still widely used in clinical practice in the operating room (OR), ICU, and in normal wards, although to a lesser extent. 
Citation: Guarnieri M, Belletti A,Saglietti F, Bignami E (2016) Central Venous Pressure as a Predictor of Fluid Responsiveness: Is This All You Need?. Gen Med (Los Angeles) 4: 228. doi:10.4172/2327-5146.1000228

Page 2 of 2

Larger well powered clinical trials might be useful to assess reliability in the prediction of fluid responsiveness during the perioperative period.

\section{References}

1. Funk DJ, Jacobsohn E, Kumar A (2013) The role of venous return in critical illness and shock-part I: physiology. Crit Care Med 41: 255-62.

2. Marik PE (2014) Iatrogenic salt water drowning and the hazards of a high central venous pressure. Ann Intensive Care 4: 21.

3. Vincent JL, Pelosi P, Pearse R, Payen D, Perel A, et al. (2015) Perioperative cardiovascular monitoring of high-risk patients: a consensus of 12 . Crit Care 19: 224.

4. Marik PE, Cavallazzi R (2013) Does the central venous pressure predict fluid responsiveness? An updated meta-analysis and a plea for some common sense. Crit Care Med 41: 1774-1781.

5. Marik PE, Monnet X, Teboul JL (2011) Hemodynamic parameters to guide fluid therapy. Ann Intensive Care 1: 1.

6. Bignami E, Belletti A, Moliterni P, Frati E, Guarnieri M, et al. (2015) Clinical practice in perioperative monitoring in adult cardiac surgery: is there a standard of care? Results from an national survey. J Clin Monit Comput.
7. Sponholz C, Schelenz C, Reinhart K, Schirmer U, Stehr SN (2014) Catecholamine and volume therapy for cardiac surgery in Germany-results from a postal survey. PLoS One 9: e103996.

8. Cannesson M, Pestel G, Ricks C, Hoeft A, Perel A (2011) Hemodynamic monitoring and management in patients undergoing high risk surgery: a survey among North American and European anesthesiologists. Crit Care 15: R197.

9. Rizza A, Bignami E, Belletti A, Polito A, Ricci Z, et al. (2016) Vasoactive Drugs and Hemodynamic Monitoring in Pediatric Cardiac Intensive Care: An Italian Survey. World J Pediatr Congenit Heart Surg 7: 25-31.

10. Siegenthaler N, Giraud R, Saxer T, Courvoisier DS, Romand JA, et al. (2014) Haemodynamic monitoring in the intensive care unit: results from a web-based Swiss survey. Biomed Res Int 2014: 129593.

11. Lansdorp B, Lemson J, van Putten MJ, de Keijzer A, van der Hoeven JG, et al. (2012) Dynamic indices do not predict volume responsiveness in routine clinical practice. Br J Anaesth 108: 395-401.

12. Saugel B, Kirsche SV, Hapfelmeier A, Phillip V, Schultheiss C, et al. (2013) Prediction of fluid responsiveness in patients admitted to the medical intensive care unit. J Crit Care 28: 537. 